\title{
microRNA-372 maintains oncogene characteristics by targeting TNFAIP1 and affects NFkB signaling in human gastric carcinoma cells
}

\author{
CHANG ZHOU* ${ }^{*}$ XIAOFENG LI* ${ }^{*}$ XIAOTING ZHANG, XIZHI LIU, \\ ZHIWEN TAN, CELI YANG and JIAN ZHANG \\ Key Laboratory of Protein Chemistry and Developmental Biology of State Education Ministry of China, \\ College of Life Science, Hunan Normal University, Changsha, Hunan 410081, P.R. China
}

Received September 19, 2012; Accepted November 23, 2012

DOI: $10.3892 /$ ijo.2012.1737

\begin{abstract}
Aberrant microRNA (miRNA) expression has been investigated in gastric cancer, which is one of the most common malignancies. However, the roles of miRNAs in gastric cancer remain largely unknown. In the present study, we found that microRNA-372 (miR-372) directly targets tumor necrosis factor, $\alpha$-induced protein 1 (TNFAIP1), and is involved in the regulation of the NFKB signaling pathway. Furthermore, overexpression of TNFAIP1 induced changes in AGS cells similar to those in AGS cells treated with miR-372-ASO. Collectively, these findings demonstrate an oncogenic role for miR-372 in controlling cell growth and apoptosis through downregulation of TNFAIP1. This novel molecular basis provides new insights into the etiology of gastric cancer.
\end{abstract}

\section{Introduction}

microRNAs (miRNAs) are approximately 22 nucleotides long and act as negative regulators of gene expression by inhibiting mRNA translation or promoting mRNA degradation (1). Hundreds of miRNAs have been identified in the human genome; bioinformatics and cloning studies have estimated that miRNAs may regulate $30 \%$ of all human genes. miRNAs can be expressed in a tissue-specific and developmental stagespecific manner, and can be involved in the regulation of embryonic stem cell fate $(2,3)$. They have attracted significant attention given their important roles in cell-cycle, apoptosis

Correspondence to: Dr Chang Zhou or Professor Jian Zhang, Key Laboratory of Protein Chemistry and Developmental Biology of State Education Ministry of China, College of Life Science, Hunan Normal University, Lushan Road, Changsha, Hunan 410081, P.R. China

E-mail: zhouchang1972@126.com

E-mail: zhangjian@hunnu.edu.cn

*Contributed equally

Key words: human gastric cancer, microRNA-372, tumor necrosis factor, $\alpha$-induced protein $1, \mathrm{NF \kappa B}$ and carcinogenesis (4). Thus, deregulation of miRNA expression may lead to a variety of disorders, including human cancer. Emerging evidence indicates that several miRNAs are deregulated in human malignancies and it has been proposed that some miRNAs may have oncogenic or tumor suppressor functions (5). For instance, the miR-17-92 cluster is markedly overexpressed in B-cell lymphomas and miR-124 has been documented as a tumor suppressor capable of inhibiting cell proliferation of glioma $(6,7)$.

Gastric cancer is one of the most common malignancies and the second leading cause of cancer-related mortality worldwide. It is estimated that 360,000 individuals succumb to gastric cancer each year in China. Despite recent advances in combining surgery, radiotherapy and chemotherapy no effective targeting therapy is available for gastric cancer, mainly due to a lack of complete understanding of the molecular mechanisms underlying gastric cancer development (8). Some miRNAs have been reported to be associated with the initiation and progression of gastric cancer. One such miRNA, miR106a, was found aberrantly expressed in gastric carcinoma tissue and was able to promote gastric carcinogenesis. The Let-7 miRNAs family as a class of tumor suppressor genes showed low levels in gastric carcinoma tissue $(9,10)$. These suggest that miRNAs are involved in gastric carcinogenesis via a variety of patterns and pathways.

Cho et al screened the expression pattern of miRNA-372 in several human gastric cancer cell lines and found that it is expressed only in the AGS cell line. miR-372 also supports the growth of the gastric cancer cell line (11). Belair et al reported that Helicobacter pylori downregulates the miR-371-372-373 cluster in proliferating gastric epithelial cells to achieve cell cycle arrest (12). The growth advantages that AGS cells gained from the high expression of the miR-371-372-373 cluster confer to them characteristics of gastric stem cell/progenitors rather than gastric mucosa differentiated epithelium. However, the molecular details of miR-372 involvement in gastric cancer development remain unclear. In the present study, we found that miR-372 was upregulated in AGS cells when compared to the undifferentiated human gastric carcinoma cell line HGC-27. Notably, overexpression of miR-372 improved HGC-27 cell growth, but inhibition of miR-372 using miR-372 inhibitor 
suppressed proliferation and increased apoptosis of AGS cells in vitro. In HGC-27 and AGS cells, we confirmed that TNFAIP1 was a target of miR-372. TNFAIP1 is an immediate-early response gene of endothelium induced by TNF $\alpha$ and IL-6 (13). It may play roles in DNA synthesis, DNA repair, cell apoptosis and human diseases $(14,15)$. TNFAIP1 was highly expressed in normal cell lines while it was lowly expressed in cancer cell lines (16). The overexpression of TNFAIP1 could accelerate the apoptosis of HeLa cells (17). Moreover, TNFAIP1 and KCTD10 suppressed the transcriptional activities of NFKB (18). We found that TNFAIP1 links miR-372 to NFкB activation, leading to increased cell survival by transactivating antiapoptotic genes downstream of NFkB. Collectively, our data indicate that an oncogenic role of miR-372 may be through control of cell growth and cell apoptosis by downregulating the TNFAIP1 and positively regulating NFkB expression.

\section{Materials and methods}

Cell culture and transfection. The human gastric carcinoma cell lines (AGS, HGC-27) and HEK293, Cos7 cell lines were obtained from the Cell Bank of the Chinese Academy of Sciences (China). Cells were cultured in DMEM (Gibco) or F12 (Gibco) media that was supplemented with $10 \%$ fetal bovine serum, $100 \mu \mathrm{g} / \mathrm{ml}$ of streptomycin and $100 \mathrm{IU} / \mathrm{ml}$ of penicillin, and maintained at $37^{\circ} \mathrm{C}$ in a humidified atmosphere with $5 \% \mathrm{CO}_{2}$. The miRNA mimics and inhibitors that were used in this study were purchased from GenePharma (Shanghai, China). All transfections were carried out using Lipofectamine 2000 (Invitrogen, USA), according to the manufacturer's instructions.

MTT assay. The AGS cells were seeded in 24-cell well plates at 16,000 cells/well. Twenty-four hours after transfection, the cells were incubated with $80 \mu \mathrm{l} \mathrm{MTT}$ at $37^{\circ} \mathrm{C}$ for another $4 \mathrm{~h}$. Then the medium was removed and the precipitated formazan was dissolved in $400 \mu \mathrm{l}$ DMSO. After shaking for $15 \mathrm{~min}$, the absorbance at $420 \mathrm{~nm}$ was detected using a microplate spectrophotometer.

FACS assays. Cells were harvested at $600 \mathrm{~g}$ for $3 \mathrm{~min}$, washed twice in PBS at room temperature and resuspended in appropriate PBS. Then, cells were resuspended in 1X FACS banding buffer containing Annexin-V and propidium iodide and analyzed using a FACS flow cytometer (BD Biosciences). A total of 20,000 cells were counted for each sample.

Plasmid construction. The 3'UTR of TNFAIP1 was amplified from HeLa cDNA using RT-PCR and was inserted into the 3 '-end of the firefly luciferase gene of the dual-luciferase miRNA target expression vector pmirGLO (Promega Corp., Madison, WI, USA) between the SacI and Xbal sites. Similarly, 3'UTR mutants, which contained mutated miR-372 binding sites, were cloned to the pmirGLO between the same sites. The primer sequences used for RT-PCR amplification are shown in Table I. All primers were synthesized by Shanghai Sangon Biotech (Sangon Biotech, China). Sequences that were inserted into the plasmid were verified by DNA sequencing. $\mathrm{pCMV}$ and $\mathrm{pLuc-NF \kappa B}$ vectors were purchased from Clontech.
Luciferase assay. The dual-luciferase reporter plasmids were co-transfected with miRNA mimics (GenePharma) into HEK 293 cells or AGS cells. At $24 \mathrm{~h}$ post-transfection, cells were assayed for luciferase activity using the Dual-Glo Luciferase assay system (Promega), according to the manufacturer's instructions. The firefly luciferase activities were normalized to Renilla luciferase activity. The firefly luciferase activity of the cells that were transfected with miRNA mimics was represented as the percentage of activity relative to that of the cells that were transfected with negative control miRNA mimics. For each transfection, the luciferase activity was averaged from three replicates (19).

RNA extraction and real-time RT-PCR analysis. Total RNA was extracted using TRIzol reagent (Invitrogen) according to the manufacturer's instructions. For miRNA detection, $2 \mu \mathrm{g}$ of small RNA was reverse transcribed to cDNA using miRNA First-Strand cDNA Synthesis kit (Invitrogen) according to the manufacturer's instructions. Quantitative real-time PCR (qRT-PCR) analysis for miR-372 was performed in triplicate with the SYBR Green PCR Master Mix (Perkin-Elmer, Applied Biosystems) according to the manufacturer's instructions. U6 RNA was used to normalize expression. To detect the target genes, $2 \mu \mathrm{g}$ of total RNA was reverse transcribed to cDNA using oligo(dT) primers and Moloney murine leukemia virus reverse transcriptase (Promega). qRT-PCR was used to determine the expression levels of TNFAIP1 using the primers presented in Table I. $\beta$-actin levels were used to normalize expression. Data analysis was performed using the $2^{-\Delta \Delta C t}$ method (20).

Immunofluorescent staining. Cells were cultured on glass coverslips in the 12-cell well. The cells were fixed using $4 \%$ paraformaldehyde, permeabilized and incubated with the primary antibodies and then with the secondary antibodies. The primary antibodies used were rabbit polyclonal anti-TNFAIP1, and mouse monoclonal anti-NFkB (P65) antibodies, while the secondary antibodies were Alexa Fluor 488 goat anti-rabbit and Alexa Fluor 488 goat anti-mouse antibodies (Molecular Probes). Hoechst 33258 (Sigma) was used to stain the nuclei. Fluorescence on the processed slips was analyzed using a fluorescent microscope (ZESS).

Western blot analysis. At $24 \mathrm{~h}$ posttransfection, cells were harvested and lysed in RIPA buffer [50 mM Tris- $\mathrm{HCl}$ (pH 7.2), $150 \mathrm{mM} \mathrm{NaCl}, 1 \%$ (v/v) Triton X-100, 1\% (w/v) sodium deoxycholate, $0.1 \%(\mathrm{w} / \mathrm{v}) \mathrm{SDS}$ ] with protease inhibitors. Proteins were separated on $10 \%$ SDS-polyacrylamide gel and transferred to PVDF membranes. A PageRuler prestained protein ladder was used as a molecular marker. Antibodies to TNFAIP1, NFאB, caspase-8, PARP, Bcl-2 or the endogenous control $\beta$-actin were incubated with the blot overnight at $4^{\circ} \mathrm{C}$. The secondary antibody was added after the membrane was washed three times with TBST. The protein was detected using a HRP-conjugated secondary antibody and a Chemilucent ECL Detection system (Millipore, Billerica, MA, USA).

Statistical analysis. Results are presented as the means \pm standard deviation from at least three separate experiments. The 
Table I. The oligonucleotides used in this study.

\begin{tabular}{|c|c|c|}
\hline Name & & Sequence $\left(5^{\prime}-3^{\prime}\right)$ \\
\hline \multirow[t]{2}{*}{ PmirGLO-TNFAIP1 } & Forward & CGAGCTCGTGCTGCCTGGGTCTCTGC \\
\hline & Reverse & GCTCTAGAGCAGCTGCTCTGTCGGATGTTT \\
\hline \multirow[t]{2}{*}{ TNFAIP1-mut1 } & Forward & CCTGCTCATGTCTGGAGAC \\
\hline & Reverse & CAGACATGAGCAGGGGCAA \\
\hline TNFAIP1-F2 & Forward & TGTGCAGAAGGGCTACTGC \\
\hline TNFAIP1-R2 & Reverse & GCAGTAGCCCTTCTGCACA \\
\hline \multirow[t]{2}{*}{ TNFAIP1-mut2 } & Forward & ATTCTCATGTACATGACAATAAG \\
\hline & Reverse & TCAGTACAACATGAGTTAAAGAA \\
\hline \multirow[t]{2}{*}{ TNFAIP1-CDS } & Forward & AGTCGACGATGTCAGGGGACACCTGT \\
\hline & Reverse & GGGTACCTCAGTCACGATGAGTGGA \\
\hline \multirow[t]{2}{*}{ RT-TNFAIP1 } & Forward & GCACTTTGGGCACCATTTTGA \\
\hline & Reverse & CGGTTCTGAGGGAGGGTGAT \\
\hline U6 & Forward & TGCGGGTGCTCGCTTCGGCAGC \\
\hline \multirow[t]{2}{*}{$\operatorname{miR}-372$} & Forward & GCCCGCAAAGTGCTGCGACAT \\
\hline & Reverse (universal) & CCAGTGCAGGGTCCGAGGT \\
\hline \multirow[t]{2}{*}{$\beta$-actin } & Forward & CCTGTACGCCAACACAGTGC \\
\hline & Reverse & ATACTCCTGCTTGCTGATCC \\
\hline
\end{tabular}

Restriction or mutation sites are underlined.

differences among groups were analyzed using the doublesided Student's t-test and $\mathrm{P}<0.05$ was considered to indicate a statistically significant difference.

\section{Results}

miR-372 regulates growth of the gastric cancer cell line. It is known that miR-372 is involved in the tumorigenesis of the testicular germ cell tumor of adolescents and adults. Cho et al (11) analyzed the miR-372 expression profile of several gastric cancer cell lines and only found the AGS cell line expressing miR-372. They also showed miR-372 plays an important role in gastric adenocarcinoma carcinogenesis. To further investigate the relationship between miR-372 and gastric adenocarcinoma, we introduced HGC-27, which is an undifferentiation gastric cancer cell to our experiment. The relative expression of miR-372 measured using real-time PCR demonstrated miR-372 expression levels in AGS are markedly higher than in HGC-27 cells, in which miR-372 was almost undetected (data not shown).

Next, we studied the growth of gastric cancer cells affected by miR-372 using MTT assay. We found that the growth rate of AGS cells transfected with the miR-372 inhibitor (miR-372-ASO) was lower than that of cells transfected with miR-NC, miR-NC-ASO and miR-372 mimics (Fig. 1A). However, the growth rate of HGC-27 cells was elevated when transfected with miR-372 mimics, compared with the growth rate of cells treated with miR-372-ASO, miR-NC-ASO and miR-NC (Fig. 1B). Our data indicate that miR-372 could affect the growth of the gastric cancer cell line.
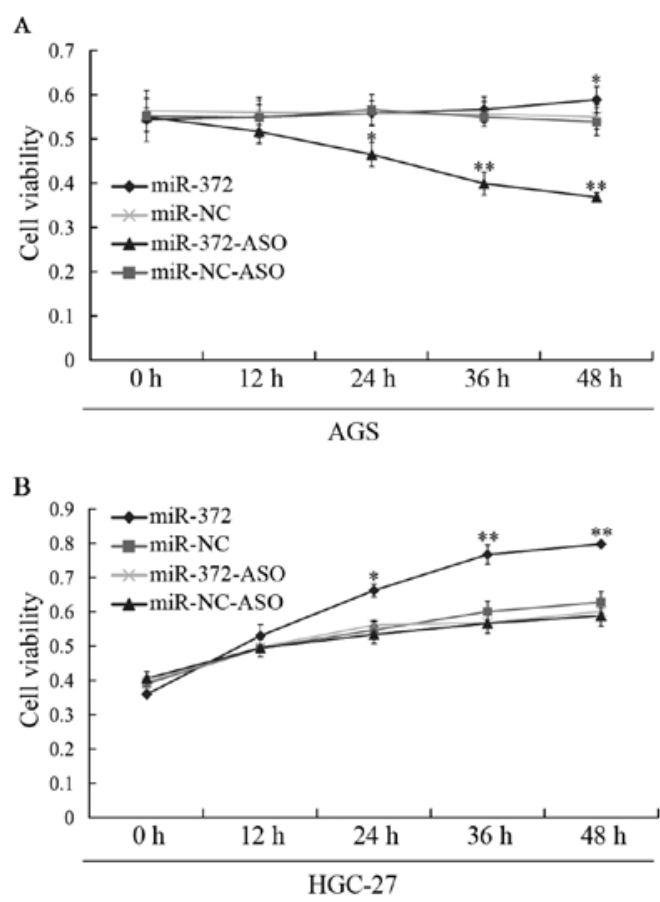

Figure 1. miR-372 regulates growth of AGS and HGC-27 gastric cancer cell lines. MTT assay of AGS cells (A) or HGC-27 cells (B) from 0 to $48 \mathrm{~h}$ after transient transfection with miR-372, miR-372 inhibitor (miR-372-ASO, fully $2^{\prime} \mathrm{O}$-methyl modified RNA oligo), miR-NC and miR-NC-ASO. The data represent the means $\pm \mathrm{SD}$ of three different experiments. ${ }^{*} \mathrm{P}<0.05$; ${ }^{* *} \mathrm{P}<0.01$.

miR-372 affects apoptosis of AGS cells. Next, we focused on AGS cells with aberrant expression of miR-372. To determine 

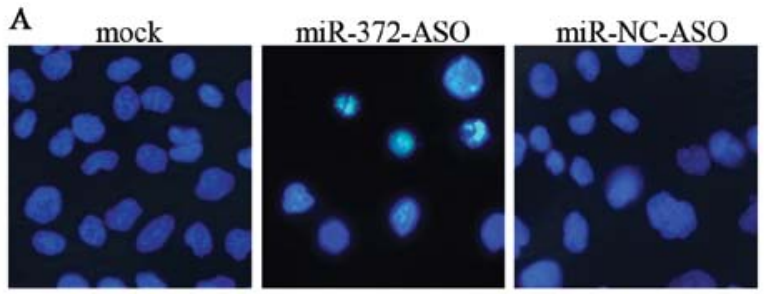

B
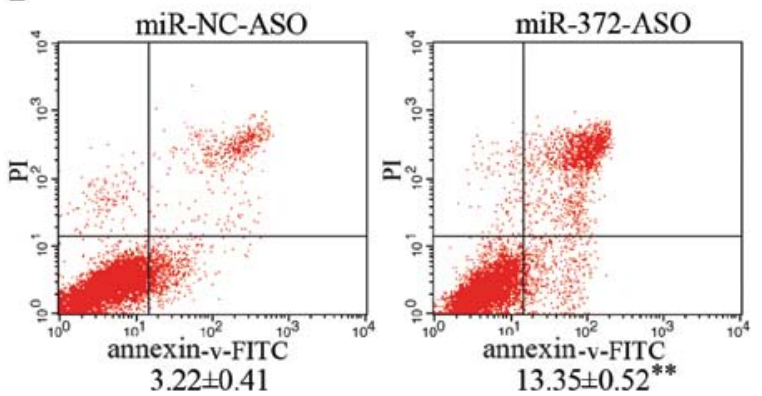

C

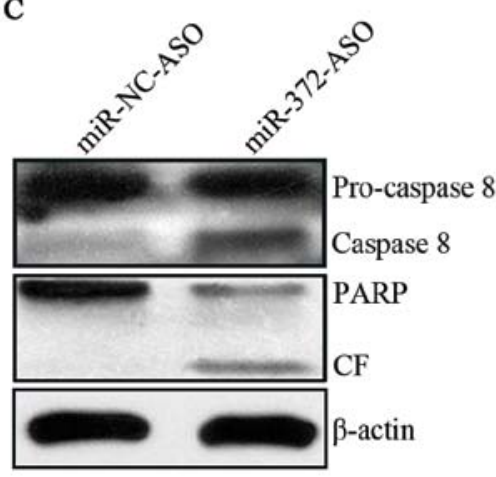

Figure 2. Cell apoptosis induced by miR-372-ASO. (A) Hoechst staining revealed AGS cell apoptosis compared to mock and miR-NC-ASO. Fortyeight hours after transfection, Hoechst staining was performed as described in Materials and methods. (B) FACS assay indicated miR-372-ASO induces AGS cell apoptosis compared to miR-NC-ASO. AGS cells were transfected with $40 \mathrm{nM} \mathrm{miR-372-ASO}$ or miR-NC-ASO, $48 \mathrm{~h}$ after transfection, apoptosis assay was performed. (C) Western blot analysis of cleaved PARP, caspase-8, and $\beta$-actin after $48 \mathrm{~h}$ of treatment with miR-372-ASO or miR-NC-ASO. The data represent the means $\pm \mathrm{SD}$ of three different experiments. ${ }^{* *} \mathrm{P}<0.01$.

whether miR-372 regulates apoptosis of AGS, we conducted Hoechst 33258 staining assay. The results indicated that suppression of miR-372 induced nuclear foci compared with mock and miR-NC-ASO (Fig. 2A). Moreover, we detected cell apoptosis in AGS cells using an Annexin-V-FITC apoptosis detection kit and found that apoptosis rates were elevated by $\sim 4$-fold in miR-372-ASO vs. miR-NC-ASO-treated cells (Fig. 2B). Further experiments revealed that miR-372-ASOtreated AGS cells induced the expression of PARP and cleaved caspase-8 (Fig. 2C), members of a general apoptosis pathway. In brief, these results indicate that miR-372 regulates the caspase- 8 apoptosis pathway and inhibits the apoptosis of AGS cells.

TNFAIPl is a target of miR-372 in AGS cells. To understand the mechanisms by which miR-372 increases AGS cell growth and inhibits apoptosis, we used several computational methods to help identify miR-372 targets in humans. We used three open access programs (TargetScan, miRanda and
picTar) to predict targets of miR-372. We found TNFAIP1 and DAZAP2 have a relative higher score and have potential to be target genes of miR-372. We then cloned the TNFAIP1 3'UTR and DAZAP2 3'UTR target sites into the PmirGLO plasmid (the predicted binding sites for miR-372 are shown in Fig. 3A). The luciferase reporter assay which was performed in HEK 293 cells indicated that the activity of the reporter containing the 3'UTR of the TNFAIP1 gene was decreased following treatment with miR-372 mimics, whereas the reporter containing the 3'UTR of DAZAP2 genes was not obviously altered (Fig. 3B). We also constructed three mutant vectors of the 3'UTR of TNFAIP1 as shown in Fig. 3C. Two single site mutation vectors, a whole site mutation vector and the luciferase reporter assay were performed with the mutated construct in the HEK293 cells. The results demonstrated that the luciferase activity was elevated in all mutated vectors and the relative luciferase activity of two single site mutation vectors increased less compared with the whole site mutation vector. Further luciferase assay was conducted in AGS cells (Fig. 3D). miR-372 showed a significant reduction of luciferase activity, with either TNFAIP1 luciferase vector or co-transfected PmirGLO-MT3 with miR-372 mimics (three gradients). The reduction of luciferase activity by miR-372 mimics could be rescued by co-transfection of miR-372 inhibitor (three gradients) with PmirGLO-MT3. Thus, these results demonstrate that $\mathrm{miR}-372$ can direct bind the two presumptive sites of TNFAIP1 3'UTR.

miR-372 regulates TNFAIPl expression at both the $m R N A$ and protein levels. To determine whether miR-372 can regulate TNFAIP1 expression in vivo, the background protein levels of AGS and HGC-27 cells were also detected (Fig. 4A). This result was consistent with the hypothesis that AGS cells are characterized by high miR-372 expression and lower TNFAIP1 protein levels, while in HGC-27 cells, miR-372 is undetected and TNFAIP1 protein levels are higher. Furthermore, overexpression of miR-372 mimics or negative control (miR-NC) was performed in HGC-27 cells. The result was that overexpression of miR-372 strongly decreased expression of TNFAIP1 at both the protein (Fig. 4B) and the mRNA level (Fig. 4D). However, when transfected with miR-372 inhibitors (miR-372-ASO) in AGS cells, which is $2^{\prime} \mathrm{Ome}$ chemically modified, single stranded nucleic acid designed to specifically bind to and inhibit endogenous miR-372 molecules, the observations indicated that TNFAIP1 is negatively regulated by miR-372 both at the protein (Fig. 4C) and mRNA levels (Fig. 4E). These results demonstrate that miR-372 negatively regulates TNFAIP1 expression.

The effect of TNFAIP1 overexpression is consistent with miR-372-ASO treated in AGS cells. To further investigate the effect of TNFAIP1 in AGS cells, we constructed TNFAIP1 expression vector pCMV-TNFAIP1, which did not contain 5' and 3'UTR of TNFAIP1. Then, pCMV-TNFAIP1 and pCMV vectors were transfected with AGS cells to study cellular mechanisms such as cell activity and apoptosis. This experiment indicated that independently overexpression of TNFAIP1 suppressed cell viability (Fig. 5A) and increased the apoptosis of AGS cells (Fig. 5B and C). We also examined the cleavage of caspase- 8 and the subsequent 
A

rat $5^{\prime} \ldots$ AGGACCCAUCUUUAA $A$ GCACUUU....3

mouse $5^{\prime}$...AGGACCCAUCUUUAAAGCACUUU....3'

human $5^{\prime}$....CCUGCCUUGCCC.......AGCACUUC....3'Position 170-192 of TNFAIP1 3'UTR

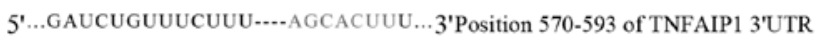

5'....ACAUCACAUACC......CAGCACUUC.... 3'Position 46-52 of DAZAP2 3' UTR

3' U G CG AGUUUACAGCGUCGUGAAA 5 'hsa-miR-372

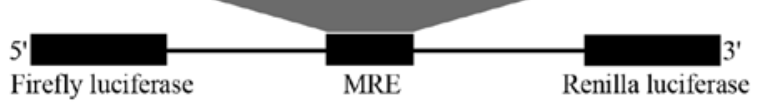

$\mathrm{C}$

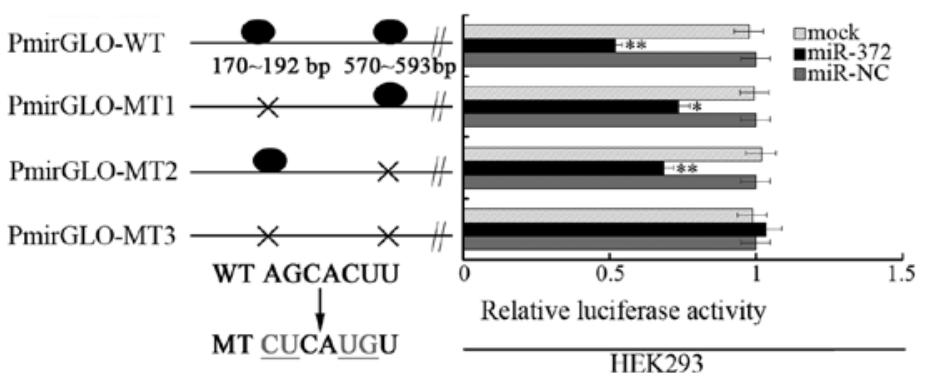

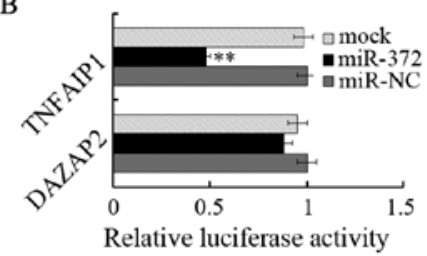

D

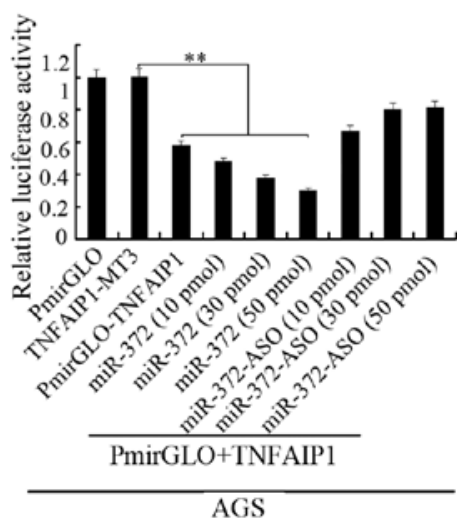

Figure 3. TNFAIP1 is one of the direct targets of miR-372. (A) Diagram of luciferase reporter constructs and the binding site of miR-372 in the predicted target sequences. (B) Luciferase assay. HEK293T cells were transiently co-transfected with luciferase reporter constructs (PmirGLO-TNFAIP1 and PmirGLODAZAP2), miR-372 or negative control miRNA. Cells transfected with empty luciferase vector were used as a mock. The relative luciferase activities were normalized against the Renilla luciferase activities. (C) Sketch of TNFAIP1 mutated vectors and luciferase assay in HEK293 cells. Cells transfected with empty luciferase vector were used as a mock. The mutated site of TNFAIP1 3'UTR is indicated in underlined italics. (D) Luciferase assay in AGS cells. AGS cells were transiently transfected with luciferase vector (PmirGLO) only (channel 1), TNFAIP1-MT3 vector (channel 2), wild-type TNFAIP1 luciferase construct (WT-TNFAIP1) (channel 3) (with three gradients) and co-transfected with miR-372 mimics (with three gradients) (channels 4-6) and inhibitor (channels 7-9) luciferase reporter constructs (WT-TNFAIP1). The relative luciferase activities were normalized against the Renilla luciferase activities. Data represent the average of three independent runs; bars, mean \pm standard error (SEM). ${ }^{*} \mathrm{P}<0.05 ;{ }^{* *} \mathrm{P}<0.01$.

A

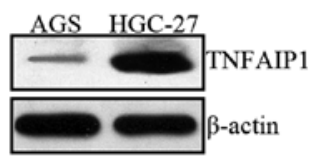

B

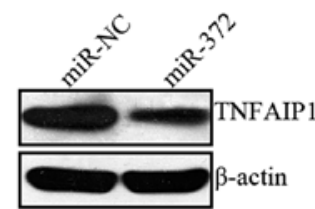

D

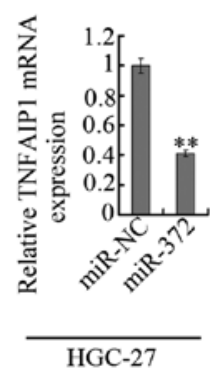

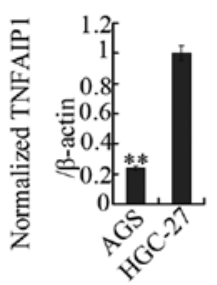

$\mathrm{C}$

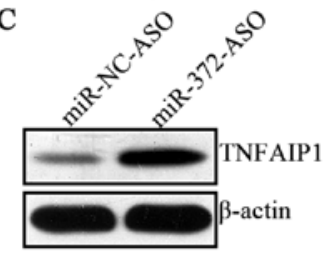

E

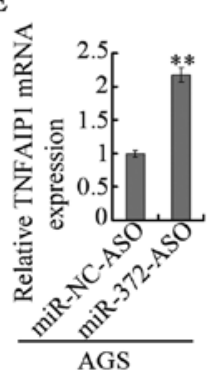

Figure 4. Effect of miR-372 on TNFAIP1 expression. (A) The background TNFAIP1 protein levels of AGS and HGC-27 cells were measured by western blotting. (B and C) HGC-27 cells were transfected with miR-372 or miR-NC, whereas AGS cells were transfected with miR-372-ASO or miRNC-ASO, respectively. (D and E) After $48 \mathrm{~h}$, cell lysates were immunoblotted for TNFAIP1 and qRT-PCR was used to detect the mRNA level of TNFAIP1. Data represent the average of three independent runs; bars, mean \pm standard error (SEM). ${ }^{* *} \mathrm{P}<0.01$.

proteolytic cleavage of PARP in AGS cells transfected with pCMV-TNFAIP1 (Fig. 5D). Western blotting assay revealed that cleavage of apical pro-caspase- 8 and PARP into the characteristic activate fragments. In brief, these results demonstrated that TNFAIP1 produces the same effect as the miR-372 inhibitor treated in AGS cells and indicates that miR-372 regulates cell growth, apoptosis of AGS cells by targeting TNFAIP1.

miR-372 regulates the expression of $N F \kappa B(p 65)$ through TNFAIP1. TNFAIP1 can be induced by TNF $\alpha$ and IL6 at the same time. The TLR family and TNF $\alpha$ receptor family possess numerous biological functions such as cell apoptosis, cell proliferation and inflammatory response and, mostly, these physiological functions are performed by activating the transcriptional activity of $\mathrm{NF} \mathrm{B}$ and then regulating downstream gene expression and TLR and TNFRs signal pathways are precisely regulated by other negative regulators in return. However, TNFAIP1 is a protein that can be induced by the tumor necrosis factor $\alpha(\mathrm{TNF} \alpha)$. Several microRNAs, such as Let-7, miR-218 and miR-199a, are related to $\mathrm{NF \kappa B}$ signaling (21). Thus, we hypothesized that miR-372 is also involved in NFKB signaling by TNFAIP1.

To further investigate the regulation network of miR-372, we detected TNFAIP1 and NFKB (p65) in miR-372 mimic transfected cells (HGC-27) (Fig. 6A) and miR-372-ASO transfected cells (AGS) (Fig. 6B). In this experiment, TNFAIP1 was inhibited by miR-372 and NFкB presented 
A

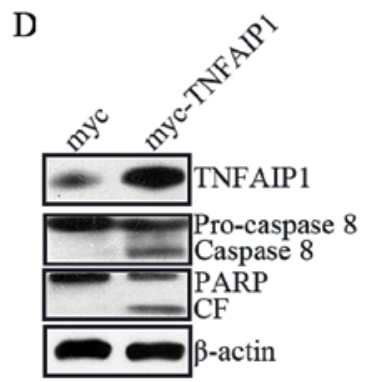

B
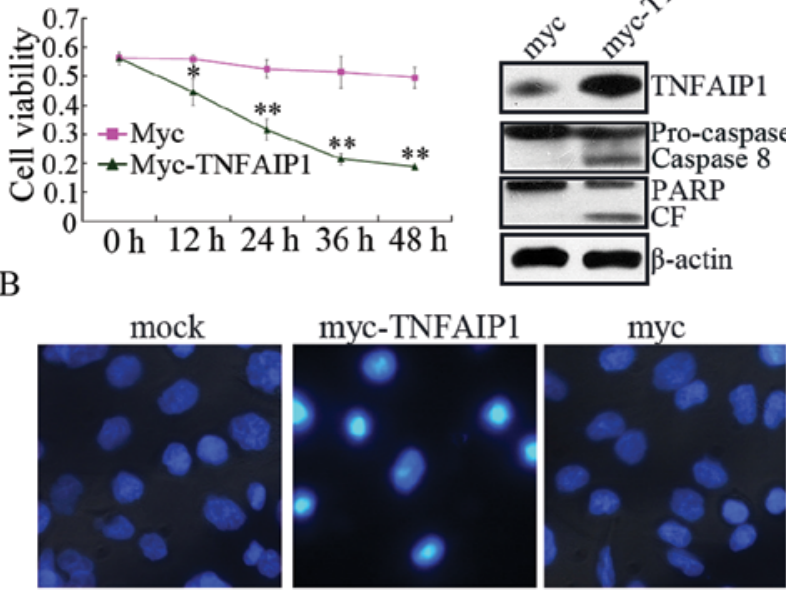

C

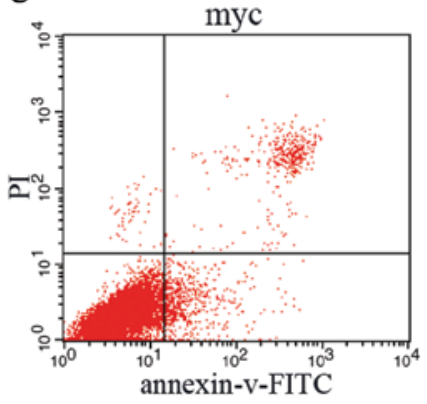

$3.84 \pm 0.45$

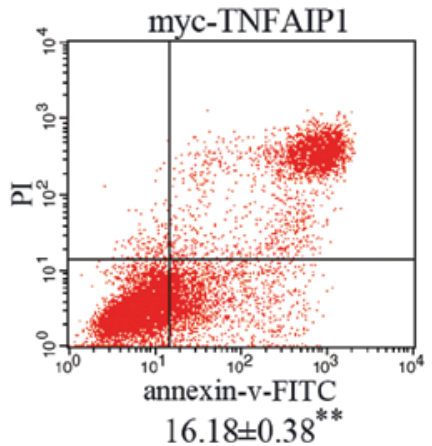

$16.18 \pm 0.38^{* *}$

Figure 5. TNFAIP1 overexpression affects the growth and apoptosis of AGS cells. AGS cells were transiently transfected with pCMV-TNFAIP1 or pCMV empty vector. After transfection for $24 \mathrm{~h}$, TNFAIP1 expression was measured by western blotting. (A) Cell growth activity was detected using MTT assay at the indicated times after transfection. At $24 \mathrm{~h}$ after transfection, the apoptosis of AGS cells was detected by Hoechst staining assay (B) and FACS analysis (C). Untreated cells were used as a mock (D) Western blot analysis of overexpression of pCMV-TNFAIP1 and cleaved PARP, caspase- 8 , and $\beta$-actin after $24 \mathrm{~h}$ of treatment with pCMV-TNFAIP1 or pCMV empty vector. The data represent the average of three independent runs; bars, mean \pm standard error $(\mathrm{SEM}) .{ }^{*} \mathrm{P}<0.05 ;{ }^{* *} \mathrm{P}<0.01$.

striking upregulation in the HGC-27 cells. On the contrary, along with TNFAIP1 expression improved by suppression of miR-372 in the AGS cells, NFkB showed downregulation and BCL-2 protein levels, which were reported to be regulated by TNFAIP1 in HeLa cells, also decreased (22). Immunofluorescence staining also revealed an increase in TNFAIP1 protein expression (Fig. 6C) and a reduction of $\mathrm{NF \kappa B}$ (p65) levels in AGS cells treated with miR-372-ASO (Fig. 6D). Furthermore, we detected NFkB luciferase activity alteration in Cos7 cells, in which TNFAIP1 expression was markedly high. We transiently transfected with luciferase empty vector (Luc) or Luc+NFKB and transiently co-transfected Luc+NFkB with miR-372 to Cos7 cells (Fig. 6E). The results demonstrated the luciferase activity of $\mathrm{NFKB}$ decreased compared to the luciferase vector (Luc) and can be reversed by the treatment of miR-372. Therefore, these findings reveal that miR-372 can regulate NFkB signal by affecting TNFAIP1.

\section{Discussion}

At present, the role of miRNAs in a variety of human malignant neoplasms has gained significant attention, with intensive investigations into the potential of inhibiting miRNAs as a novel approach to treatment. However, how these miRNA molecules contribute to the pathogenesis of cancer remains largely unknown. A detailed study on the biological functions of the miRNA-mRNA interaction network is therefore urgently required.

The miR-372 gene is located on genomic chromosome at $19 \mathrm{q} 13.42$. The functions of miR-372 have yet to be fully explored and its relevance to carcinogenesis remains unclear. Previous studies showed that miR-372 enhances cell proliferation, stimulates cell cycle progression, or decreases apoptosis in testicular tumor germ cells and in gastric cancer cells. By contrast, miR-372 suppresses cell growth in human cervical cancer (23). Considering that the gene expression profile is tumor-specific and that the influence of miRNA alterations might strongly depend on tumor context, these results are expected.

We analyzed the proliferation rate of gastric cancer cells transfected with miR-372 or anti-miR-372 to identify its oncogenic property. Our results showed that the proliferation capacity was repressed after downregulation of miR-372 in gastric cancer AGS cell proliferation, whereas the overexpression of miR-372 promoted gastric cancer HGC-27 cell survival rate, indicating that miR-372 might be a potential growth factor for gastric cancer cells. Furthermore, we found that miR-372 decreases apoptosis in gastric cancer cells.

Since the impact of cancer miRNAs on cancer biology depends on the functions of the downstream targets they suppress, we need to discover the targets of each miRNA. It is well known that miRNAs can regulate the gene expression of multiple targets to perform pleiotropic functions $(24,25)$. According to PicTar, miR-372 has 571 predicted targets in humans. We found, through several databases, numerous important candidate targets, among which TNFAIP1 is a direct target of miR-372 in the control of gastric cancer cell proliferation $(26,27)$. TNFAIP1 is a protein which can be induced by TNF $\alpha$ and IL-6. It also relates to DNA synthesis, DNA repair, cell apoptosis and human diseases. In the present study, using a luciferase reporter system and expression assay, we confirmed that TNFAIP1 is negatively regulated by miR-372. When we examined the relationship between TNFAIP1 mRNA and miR-372 in gastric cancer cell lines, an inverse correlation between miR-372 and the TNFAIP1 protein expression was noted by western blot analysis and immunofluorescence (Fig. 4C). TNFAIP1 was shown to be expressed at a high level in normal cells and was downregulated in several cancerderived cell lines. miR-372 overexpression conferred cell resistance to apoptosis, and the increased resistance correlated with the level of TNFAIP1. Conversely, reducing miR-372 expression by miRNA inhibitors greatly sensitized cells to apoptosis. miR-372 may play a key role in tumorigenesis by conferring cells resistance to apoptosis.

In recent years, several miRNAs have been found to be involved in $\mathrm{NF} \kappa \mathrm{B}$ signaling by targeting $\mathrm{NF \kappa B}$ regulators or effectors and participating in positive or negative feedback loops. In this study, we found that miR-372 is also associated 
A
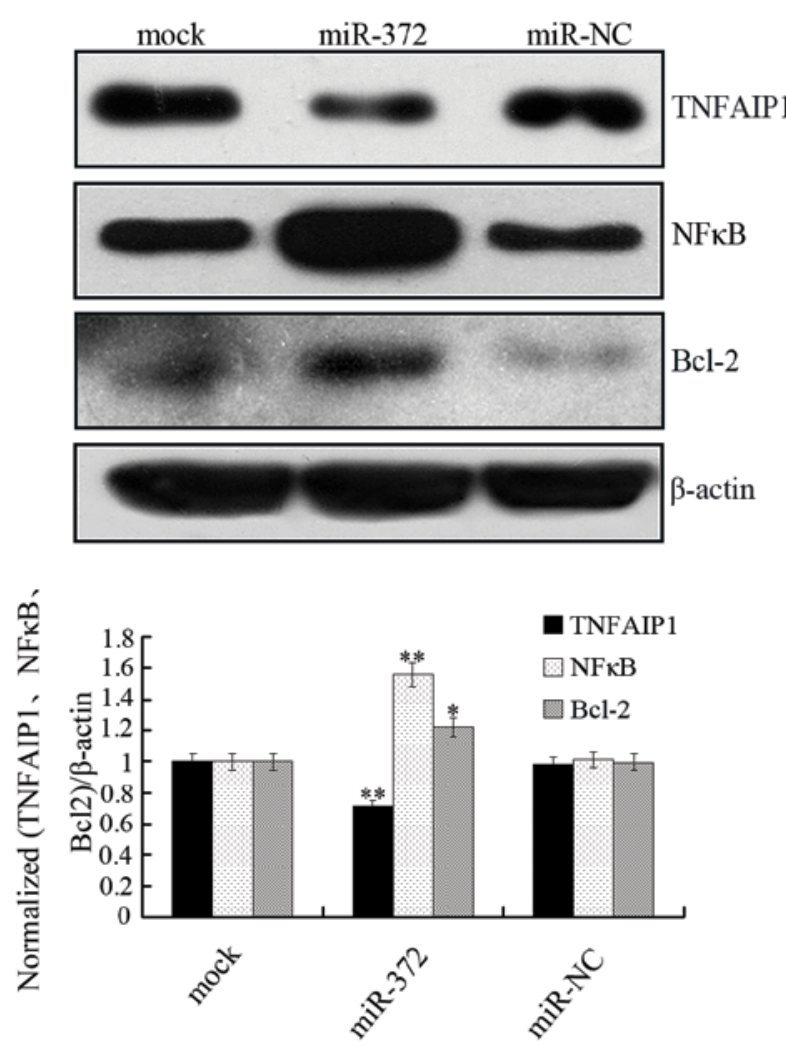

HGC-27

C

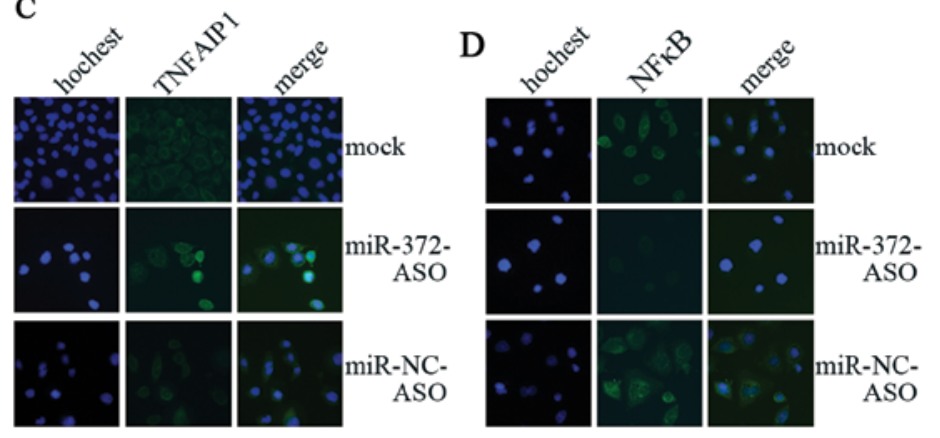

AGS
B
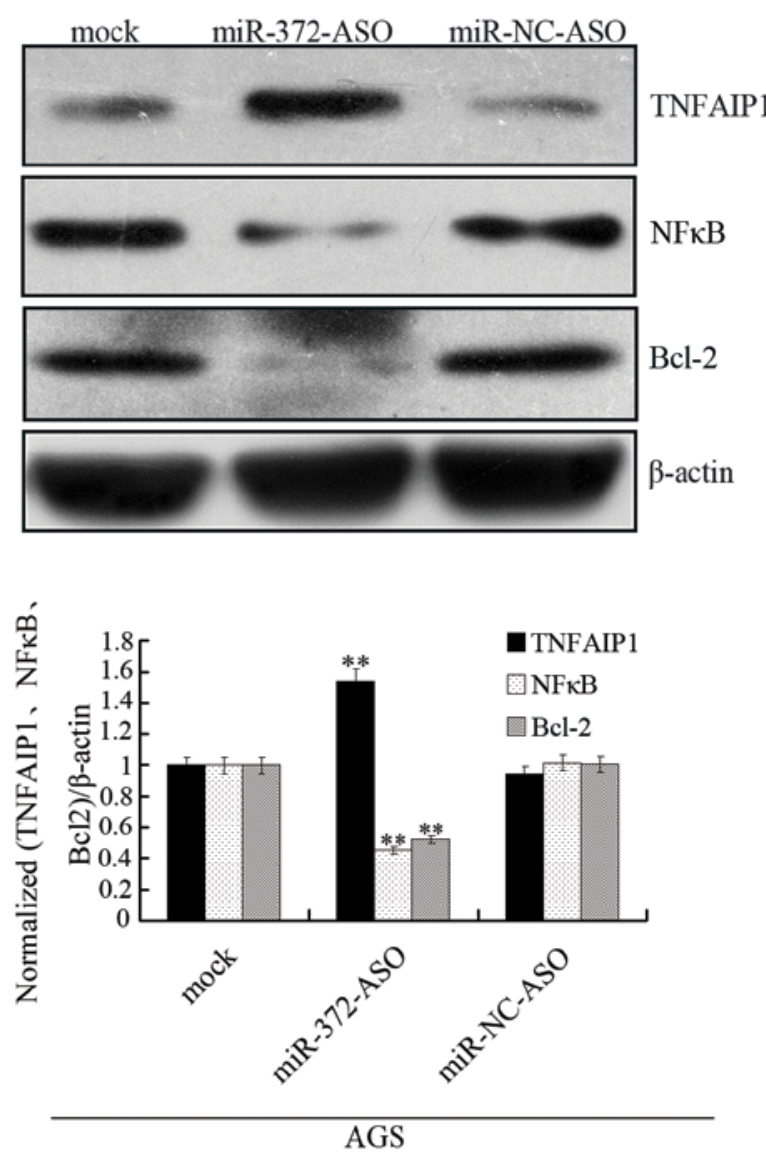

E

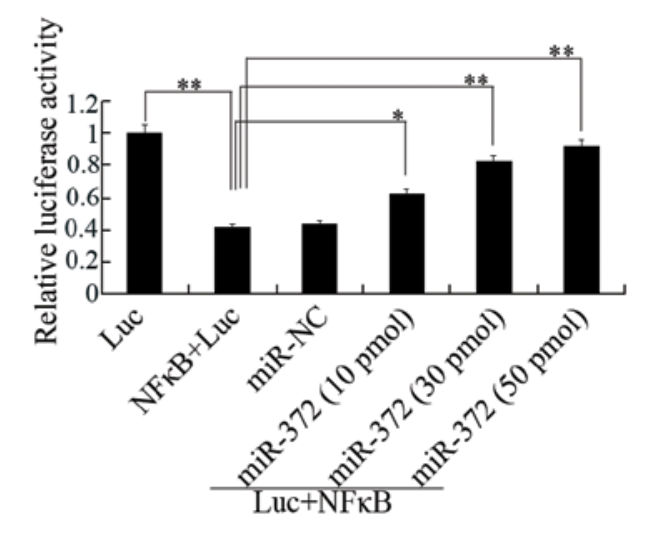

$\operatorname{Cos} 7$

Figure 6. miR-372 affects NFkB signaling by targeting TNFAIP1. (A and B) Western blotting was used to measure the expression levels of TNFAIP1, NFkB and Bcl-2 in HGC-27 and AGS cells. $\beta$-actin was used as a loading control. (C and D) TNFAIP1 and NFkB expression were determined by immunofluorescence after treatment with miR-372 inhibitor (miR-372-ASO) in AGS cells. (E) Luciferase assay of NFKB in Cos7 cells. Cos7 cells were transiently transfected with luciferase vector (Luc) only (channel 1), Luc+NFKB vector (channel 2) and co-transfected with Luc+NFkB with miR-372 mimics (with three gradients) (channels 3-6). The relative luciferase activities were normalized against the Renilla luciferase activities. Data represent the average of three independent runs; bars, mean \pm standard error (SEM). ${ }^{*} \mathrm{P}<0.05 ;{ }^{* * *} \mathrm{P}<0.01$.

with NFkB. When miR-372 overexpression was performed in HGC-27 cells, NFkB expression was increased with the inhibition of TNFAIP1. While miR-372 was inhibited in AGS cells, NFKB presented downregulation accompanied by increased TNFAIP1. Furthermore, NFאB transcription luciferase assay in Cos7 cells indicated the increase of luciferase activity related to the expression of TNFAIP1. Therefore, TNFAIP1 regulated by miR-372 may finally have an effect on $N F \kappa B$ signaling and the miRNAs which converged with the NFאB network may be enlarged. The oncogenic properties of miR-372 may partly derive from increased NFKB (Fig. 7). Furthermore, although we have shown that miR-372 can target TNFAIP1 and affect 


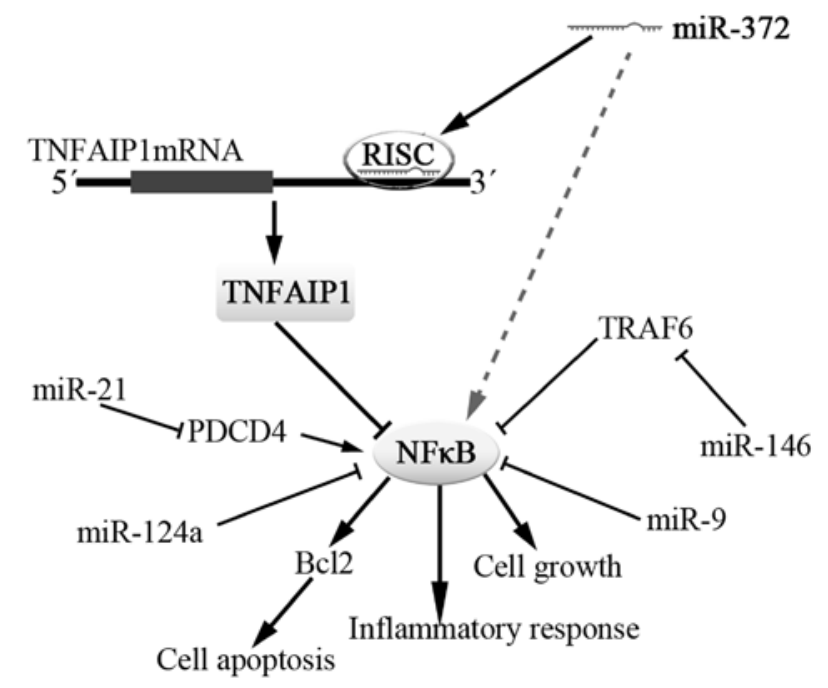

Figure 7. Schematic model showing the mechanism through which miR-372 regulates TNFAIP1 and NFKB signaling.

NFKB signaling, our laboratory recently demonstrated that TNFAIP1 suppressed the transcriptional activities of NFkB. However, the detailed action between TNFAIP1 and NFKB may need further study as well as the reason why miR-372 was overexpressed in AGS cells. Our findings demonstrate that miR-372 enhances cell proliferation, or decreases apoptosis in gastric cancer cells via directly regulating the expression of TNFAIP1, suggesting that miR-372 can serve as a potential target in the treatment of gastric cancer. We have shown that miR-372 affects NFkB and that these effects may be achieved by regulating the expression of TNFAIP1. This may, in part, be the reason for miR-372 possessing oncogenic properties.

\section{Acknowledgements}

This study was supported by the National Natural Science Foundation of China (grant nos. 81071696, 31071150 and 30900827), the Hunan Provincial Innovation Foundation for Postgraduate (CX2012B227) and the Project of Chang Sha Science and Technology Plan (K1109006-31).

\section{References}

1. Bartel DP: MicroRNAs: genomics, biogenesis, mechanism, and function. Cell 116: 281-297, 2004.

2. Berezikov E, Thuemmler F, Van Laake LW, et al: Diversity of microRNAs in human and chimpanzee brain. Nat Genet 38: 1375-1377, 2006.

3. Wienholds E and Plasterk RHA: MicroRNA function in animal development. FEBS Lett 579: 5911-5922, 2005.

4. Carleton M, Cleary MA and Linsley PS: MicroRNAs and cell cycle regulation. Cell Cycle 6: 2127-2132, 2007.

5. Chen CZ: MicroRNAs as oncogenes and tumor suppressors. N Engl J Med 353: 1768-1771, 2005.
6. Inomata M, Tagawa H, Guo YM, Kameoka Y, Takahashi N and Sawada K: MicroRNA-17-92 down-regulates expression of distinct targets in different B-cell lymphoma subtypes. Blood 113: 396, 2009

7. Silber J, Lim D, Petritsch C, et al: miR-124 and miR-137 inhibit proliferation of glioblastoma multiforme cells and induce differentiation of brain tumor stem cells. BMC Med 6: 14, 2008.

8. Gao C, Zhang Z, Liu W, Xiao S, Gu W and Lu H: Reduced microRNA-218 expression is associated with high nuclear factor kappa B activation in gastric cancer. Cancer 116: 41-49, 2010

9. Motoyama K, Inoue H, Nakamura Y, Uetake H, Sugihara K and Mori M: Clinical significance of high mobility group A2 in human gastric cancer and its relationship to let-7 microRNA family. Clin Cancer Res 14: 2334-2340, 2008.

10. Xiao B, Guo J, Miao Y, et al: Detection of miR-106a in gastric carcinoma and its clinical significance. Clin Chim Acta 400: 97-102, 2009.

11. Cho WJ, Shin JM, Kim JS, et al: miR-372 regulates cell cycle and apoptosis of ags human gastric cancer cell line through direct regulation of LATS2. Mol Cells 28: 521-527, 2009.

12. Belair C, Baud J, Chabas S, et al: Helicobacter pylori interferes with an embryonic stem cell micro RNA cluster to block cell cycle progression. Silence 2: 1-16, 2011.

13. Wolf F, Marks R, Sarma V, et al: Characterization of a novel tumor necrosis factor-alpha-induced endothelial primary response gene. J Biol Chem 267: 1317-1326, 1992.

14. Link CD, Taft A, Kapulkin V, et al: Gene expression analysis in a transgenic Caenorhabditis elegans Alzheimer's disease model. Neurobiol Aging 24: 397-413, 2003.

15. Yang L, Liu N, Hu X, et al: CK2 phosphorylates TNFAIP1 to affect its subcellular localization and interaction with PCNA. Mol Biol Rep 37: 2967-2973, 2010.

16. Yang LP, Zhou AD, Li H, et al: Expression profile in the cell lines of human TNFAIP1 gene. Yi Chuan 28: 918-922, 2006.

17. Kim DM, Chung KS, Choi SJ, et al: RhoB induces apoptosis via direct interaction with TNFAIP1 in HeLa cells. Int J Cancer 125: 2520-2527, 2009.

18. Hu X, Yan F, Wang F, et al: TNFAIP1 interacts with KCTD10 to promote the degradation of KCTD10 proteins and inhibit the transcriptional activities of NF-kappaB and AP-1. Mol Biol Rep 39: 9911-9919, 2012.

19. Zeng Y and Cullen BR: Sequence requirements for micro RNA processing and function in human cells. RNA 9: 112-123, 2003.

20. Livak KJ and Schmittgen TD: Analysis of relative gene expression data using real-time quantitative PCR and the $2^{-\Delta \Delta C_{\mathrm{T}}}$ method. Methods 25: 402-408, 2001.

21. Ma X, Becker Buscaglia LE, Barker JR and Li Y: MicroRNAs in NF-kappaB signaling. J Mol Cell Biol 3: 159-166, 2011.

22. Hu W, Xie J, Zhao J, Xu Y, Yang S and Ni W: Involvement of Bcl-2 family in apoptosis and signal pathways induced by cigarette smoke extract in the human airway smooth muscle cells. DNA Cell Biol 28: 13-22, 2009.

23. Tian RQ, Wang XH, Hou LJ, et al: MicroRNA-372 is downregulated and targets cyclin-dependent kinase 2 (CDK2) and cyclin A1 in human cervical cancer, which may contribute to tumorigenesis. J Biol Chem 286: 25556-25563, 2011.

24. Alvarez JP, Pekker I, Goldshmidt A, Blum E, Amsellem Z and Eshed Y: Endogenous and synthetic microRNAs stimulate simultaneous, efficient, and localized regulation of multiple targets in diverse species. Plant Cell 18: 1134-1151, 2006.

25. Valastyan S, Reinhardt F, Benaich N, et al: A pleiotropically acting microRNA, miR-31, inhibits breast cancer metastasis. Cell 137: 1032-1046, 2009.

26. Gillis AJ, Stoop HJ, Hersmus R, et al: High-throughput microRNAome analysis in human germ cell tumours. J Pathol 213: 319-328, 2007.

27. Subramanyam D, Lamouille S, Judson RL, et al: Multiple targets of miR-302 and miR-372 promote reprogramming of human fibroblasts to induced pluripotent stem cells. Nat Biotechnol 29: 443-448, 2011. 